Case Report Journal of Epilepsy Research pISSN 2233-6249 / eISSN 2233-6257

Received November 3, 2011 Accepted November 21, 2011

Corresponding author: Tae-Sung Ko Department of Pediatrics, Asan Medical Center, University of Ulsan College of Medicine, 388-1 Pungnap-dong, Songpa-gu, Seoul 138-736, Korea

Tel. +82-2-473-3725

Fax. $+82-2-3010-3388$

E-mail; tsko@amc.seoul.kr

\section{Staged Total Callosotomy for Lennox-Gastaut Syndrome: A Case Report}

\author{
Eun Hye Lee, MD', Mi-Sun Yum, MD², Seok-Ho Hong, MD³, Jeong-Kyo Lee, MD³, \\ Su Jeong You, MD', Tae-Sung Ko, MD² \\ ${ }^{1}$ Department of Pediatrics, College of Medicine, Kyung Hee University; ${ }^{2}$ Department of Pediatrics, Asan Medical \\ Center, Children's Hospital, University of Ulsan College of Medicine; ${ }^{3}$ Department of Neurosurgery, Asan Medical \\ Center, University of Ulsan College of Medicine; ${ }^{4}$ Department of Pediatrics, Epilepsy Center, Sanggye Paik \\ Hospital, Inje University College of Medicine, Seoul, Korea
}

\begin{abstract}
We report a case with Lennox-Gastaut syndrome (LGS) who underwent staged total callosotomy with a favorable outcome. A 6-year-old boy began having myoclonic seizures at the age of 8 months and was diagnosed with LGS when he was 27 months old. Various antiepileptic drugs and a ketogenic diet failed to control his multiple types of seizures. At the age of 36 months, he underwent corpus callosotomy and achieved an immediate, post-operative seizure free state. However, 3 months later, various seizures relapsed and were refractory to additional vagus nerve stimulation. Remaining callosal fibers in the splenium noted on post-operative diffusion tensor imaging made us to perform a second operation, total callosotomy. The patient finally achieved a seizure-free state with electroencephalography (EEG) normalization noted after the staged total callosotomy. (2011;1:71-73)
\end{abstract}

Key words: Lennox-Gastaut syndrome; Callosotomy
Lennox-Gastaut syndrome (LGS) is a difficult-to treat type of epilepsy characterized by multiple types of seizures, generalized slow spike and wave activities or generalized paroxysmal fast activities in EEG, and progressive cognitive deterioration. ${ }^{1}$ Because medical treatment usually fails to achieve satisfactory seizure control, multimodal management is often required. Surgery for this condition includes callosotomy, vagus nerve stimulation or resective surgeries for limited cases with focal lesions., ${ }^{2,3}$ Among them, corpus callosotomy is effective especially for treating drop attacks which are the most notorious type of seizures in LGS. ${ }^{4,5}$ In some medical centers, anterior callostomy is often performed in order to avoid the complications of a disconnection syndrome, however, approximately $30 \%$ of these patients experience relapse of their seizures. ${ }^{6}$ In those cases, a second total callosotomy can be a useful treatment option. We report here the case of a patient with LGS who underwent staged total callosotomy, after which he achieved a seizure- free state.

\section{Case Report}

A 6 year-old boy was born at 34 weeks' gestation to healthy parents with birth weight of 3,540 $\mathrm{g}$ after an uneventful pregnancy. He had no family history of epilepsy or focal neurologic sign, but he had delayed development in fine motor and language areas since before 12 months of age. His first seizures, myoclonic seizures, occurred at the age of 8 months, and he was started on antiepileptic drugs (AEDs). At the time of his admission to our hospital at the age of 27 months, he was suffering from multiple types of seizures including drop attacks, generalized tonic seizures, and atypical absence seizures. His EEG showed generalized slow spike and wave discharges with multifocal spikes (Figure 1), and he was diagnosed as having LGS. Brain magnetic resonance imaging (MRI) showed no abnormalities. Despite various antiepileptic drugs (valproate, topiramate, zonisamide, and clobazam) and a ketogenic diet, his seizures remained uncontrolled. Long term video EEG monitoring for 48 hours showed a dozen seizures a day. The seizure semiology consisted of suddenly dropping his head with losing his tone, thus suggesting myoclonic-atonic seizures. Ictal EEG showed diffuse generalized bursts of polyspike discharges with very brief electromyographic activities. His social quotient was reported to be 27. At the age of 36 months, he underwent anterior $2 / 3$ corpus callosotomy. He achieved a post-operative seizure-free state for 3 


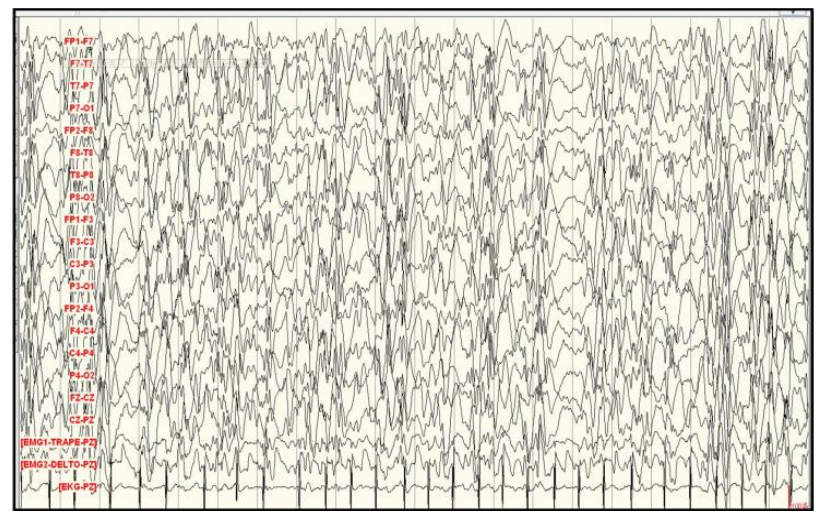

Figure 1. Interictal electroencephalogram (EEG) shows generalized slow spike and wave discharges.

months, after which he developed a relapse of his seizures. EEG performed at post-operative 10 months revealed frequent generalized spike and wave discharges and generalized paroxysmal fast activities. His seizures did not respond to either additional vagus nerve stimulation or to medical treatment at that time. As diffusion tensor imaging showed remaining callosal fibers in the splenium, we decided to perform a second total callosotomy (Figure 2). After the second total callosotomy, the patient maintained a seizure-free state for 27 months and with EEG normalization (Figure 3). He is still on the medication of valproic acid, topiramate, zonisamide, and clobazam. Developmental test performed after the second surgery showed no remarkable progress by that time. However, according to his parents, his cognitive function is improving compared to that of his pre-operative state.

\section{Discussion}

Corpus callosotomy is a palliative surgical procedure for patients with intractable seizures who are not amenable to focal resection. Since Van Wagen and Herren first performed commisurotomy in human in 1940, it is currently generally accepted that callosotomy is effective for disabling generalized seizures, particularly drop attacks. ${ }^{4,6-8}$ In drop attacks, $80-92 \%$ of patients who underwent corpus callosotomy achieved $>50 \%$ seizure reduction, and $50-61 \%$ had $>50 \%$ seizure reduction in generalized tonic clonic seizures. ${ }^{4}$ The rationale underlying diminishing seizures by corpus callosotomy is based on the hypothesis that corpus callosum is the largest commisure interconnecting the two hemispheres and that it has an important role in spreading interhemispheric epileptic activity. ${ }^{5}$ The anterior callosum carries fibers from the premotor, motor,
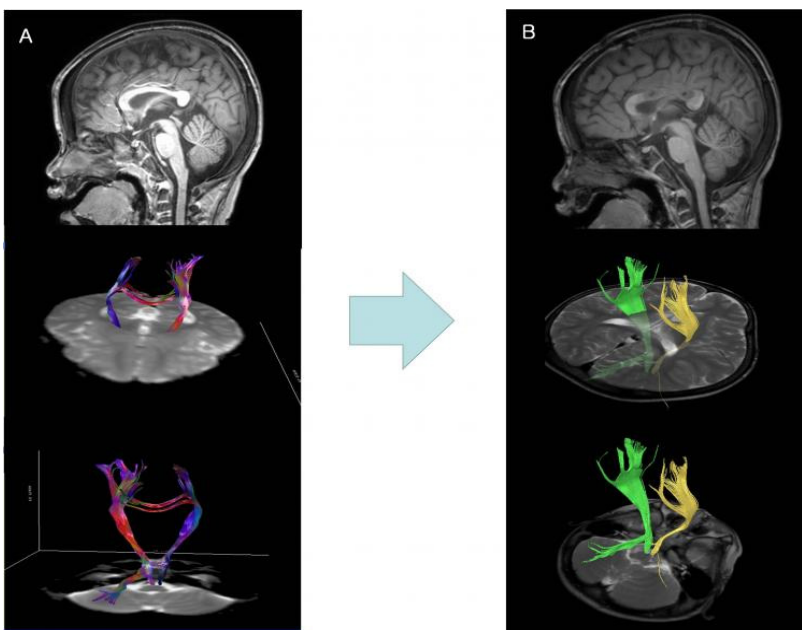

Figure 2. Pre-operative (A) and post-operative (B) sagittal T1 weighted image and diffusion tensor imaging, both showing remaining corpus callosum at genu and splenium after the first corpus callosotomy.

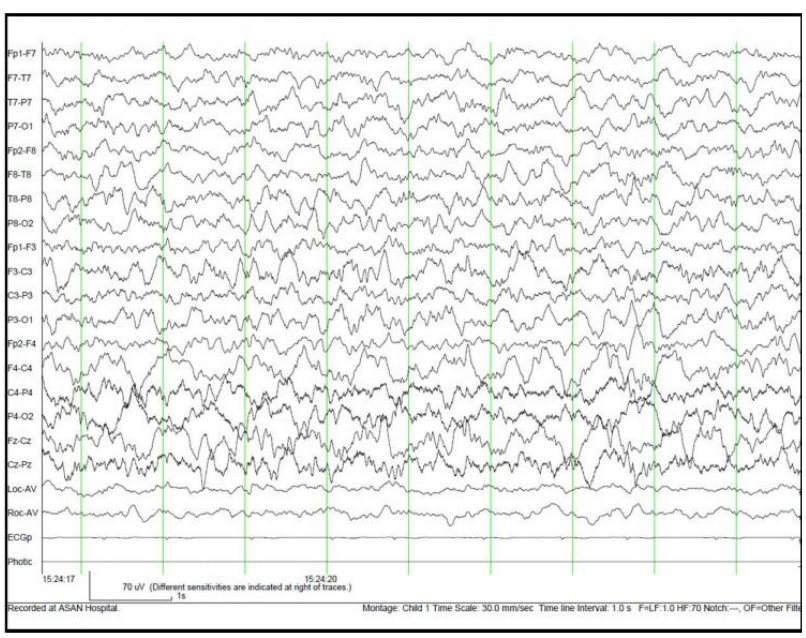

Figure 3. Follow-up electroencephalogram (EEG) obtained 4 months after the second corpus callosotomy, shows normal background activity without epileptiform discharges.

anterior insula, and anterior cingulated gyrus, that is essential for the generalization of tonic and tonic-clonic seizures, and atonic seizures. ${ }^{4,5}$ On the other hand, splneium, the posterior callosum, connects fibers from the superior parietal lobe and occipital cortex that transfers perceptual information. Therefore, total callosotomy including splenium has a greater risk of complications, such as disconnection syndrome, due to the disruption of the transfer of visual, tactile, and auditory information. ${ }^{9}$

According to the previous studies, the optimal range of callosal section has not been clearly determined. Spencer et al..$^{10}$ reported that anerior callosotomy was effective for secondarily generalized 
seizures in $35 \%$, whereas total callosotomy was effective in $77 \%$. Sunaga et al. ${ }^{6}$ also demonstrated that control of drop attacks was achieved in $76 \%$ of their patients with total callosotomy and in $54 \%$ of those with anterior callosotomy. They also reported that patients with anterior callosotomy had a higher relapse rate than patients with total callosotomy (31\% vs. $7 \%$ ). However, Mamelak et al. ${ }^{11}$ reported that anterior $1 / 2-2 / 3$ callosotomy is nearly as effective as extensive total callosotomy for reducing both drop attacks and generalized tonic clonic seizures. They proposed that the extent of callosotomy is not an important factor if at least $50-65 \%$ of the callosum is divided.

Considering the seizure outcome and the risk of complications, some centers prefer to perform an anterior callosotomy which first spares the splenium, and then to perform total callosotomy as a second step if the first surgery is not successful. ${ }^{5,9,11}$ In a report by Spencer et $a / .^{10}$ for 14 patients who failed to improve after anterior callosotomy and then underwent further total callosotomy, dramatic responses were noted for all seizure types. In Korea, Kim et al. ${ }^{9}$ have reported that a second total callosotomy performed in 4 patients who did not have clinical improvement after their first surgery, allowed 3 of them to become seizure-free and allowed $>75 \%$ seizure reduction in the other patient. We suggest that staged total callosotomy is a valid treatment option for patients with LGS suffering from drop attacks.

It is interesting that the EEG performed at 4 months post-operatively showed completely normal findings in our report. This finding supports the hypothesis that corpus callosum has a role in generating epileptiform discharges. Matsuo et al. ${ }^{12}$ performed a quantitative analysis of EEGs in patients who underwent corpus callosotomy; they found that corpus callosotomy decreased the total amount of spike discharge and the duration of burst activity in addition to disrupting bilateral synchrony. They suggested that corpus callosum not only transfers epileptic activities but also has a mutually facilitatory effect that enhances the epileptogenic state of both hemispheres. However, the precise role of the corpus callosum in epileptogenesis remains to be determined.
Here we report the case of a patient who experienced relapse of seizures after anterior callosotomy but finally obtained freedom from seizures with EEG normalization after the second total callosotomy.

We, therefore, suggest that although surgery can initially be limited to the anterior $2 / 3$ of the corpus callosum, if the first operation is unsuccessful, further total callosotomy should be considered.

\section{References}

1. Angeles DKF. Proposal for revised clinical and electroencephalographic classification of epileptic seizures. Epilepsia 1981;22:489-501.

2. You SJ, Kang HC, Ko TS, et al. Comparison of corpus callosotomy and vagus nerve stimulation in children with Lennox- Gastaut syndrome. Brain Dev 2008:30:195-9.

3. You SJ, Lee JK, Ko TS. Epilepsy surgery in a patient with Lennox-Gastaut syndrome and cortical dysplasia. Brain Dev 2007;29:167-70.

4. Asadi-Pooya AA, Sharan A, Nei M, Sperling MR. Corpus callosotomy. Epilepsy Behav 2008;13:271-8.

5. Wong TT, Kwan SY, Chang KP, et al. Corpus callosotomy in children. Child Nerv Syst 2006;22:999-1011.

6. Sunaga S, Shimizu H, Sugano H. Long-term follow-up of seizure outcomes after corpus callosotomy. Seizure 2009;18:124-8.

7. Rossi G, Colicchio G, Marchese E, Pompucci A. Callosotomy for severe epilepsies with generalized seizures: outcome and prognostic factors. Acta Neurochirurgica 1996; 138:221-7.

8. Tanriverdi T, Olivier A, Poulin N, Andermann F, Dubeau F. Long-term seizure outcome after corpus callosotomy: a retrospective analysis of 95 patients. J Neurosurgery 2009; 110:332-42.

9. Kim DS, Yang KH, Kim TG, et al. The surgical effect of callosotomy in the treatment of intractable seizure. Yonsei Med J 2004;45:233-40.

10. Spencer SS, Spencer DD, Sass K, Westerveld M, Katz A, Mattson R. Anterior, total, and two-stage corpus callosum section: differential and incremental seizure responses. Epilepsia 1993;34:561-7.

11. Mamelak AN, Barbaro NM, Walker JA, Laxer KD. Corpus callosotomy: a quantitative study of the extent of resection, seizure control, and neuropsychological outcome. J Neurosurgery 1993;79:688-95.

12. Matsuo A, Ono T, Baba H, Ono K. Callosal role in generation of epileptiform discharges: quantitative analysis of EEGs recorded in patients undergoing corpus callosotomy. Clin Neurophysio/2003;14:2165-71. 